\title{
Crystal structure of the low temperature phase of bis(butylamine) zirconium bis(nitrilotriacetate) dihydrate, $\left[\mathrm{NH}_{3}\left(\mathrm{CH}_{2}\right)_{4}\right]_{2} \mathrm{Zr}\left[\mathrm{N}\left(\mathrm{CH}_{2} \mathrm{COO}\right)_{3}\right]_{2} \cdot 2 \mathrm{H}_{2} \mathrm{O}$
}

\author{
E. Haussühl ${ }^{*, \mathrm{I}, \mathrm{Il}}$, G. Giester ${ }^{\mathrm{l}}$ and E. Tillmanns ${ }^{\mathrm{I}}$ \\ I Universität Wien, Institut für Mineralogie und Kristallographie, Althanstr. 14 - Geozentrum, A-1090 Wien, Austria \\ II present address: ETH Zürich, Laboratorium für Kristallographie, Sonneggstr. 5, CH-8092 Zürich, Switzerland
}

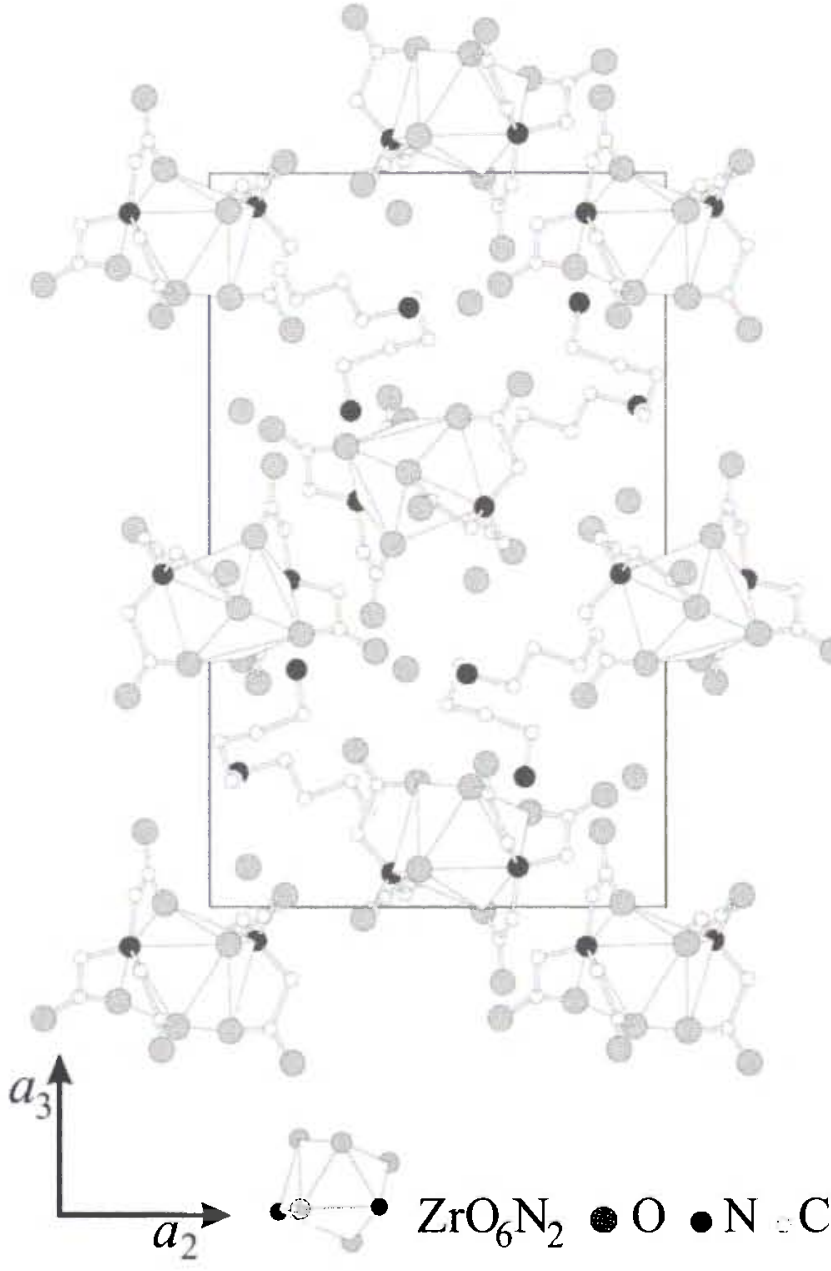

\section{Source of material}

The raw material was obtained by fractional crystallization of the reaction product of butylamine, nitrilotriacetic acid and $\mathrm{ZrCl}_{4}$ in a stoichiometric ratio at $300 \mathrm{~K}$.

\section{Discussion}

From differential scanning calorimetry runs, thermal expansion and elastic behaviour a first-order phaese transition at about 188 $\mathrm{K}$ was detected. Other distinct anomalies were found in respect to the variation of the thermoelastic constants with temperature and the attenuation of ultrasonic waves [1]. The structures of most zirconium nitrilotriacetates, so far investigated, and also of bis(butylamine) zirconium bis(nitrilotriacetate) dihydrate, are built up from puckered layers of $\mathrm{Zr}\left[\mathrm{N}\left(\mathrm{CH}_{2} \mathrm{COO}\right)_{3}\right]^{2-}$ anions with intralayer and interlayer Coulomb interactions between anions and cations and in addition strong hydrogen bonds. The anions, which $\mathrm{Zr}$ is coordinated by six oxygen and two nitrogen atoms of the nitrilotriacetate group, possess an almost perfectly persistent conformation, which has been thoroughly studied by Howard et al. [2]. In this case the intralayer bonds consist mainly of Coulomb interactions between the anions and the $\mathrm{NH}_{3}$ groups, further strengthened by hydrogen bonds between the nitrogen atoms of the $\mathrm{NH}_{3}$ groups and oxygen atoms of the carboxylic groups. The layers are held together by Coulomb interactions between $\mathrm{NH}_{3}$ groups and anions belonging to adjacent layers. These are reinforced by hydrogen bonds between the water molecules W1, W2 and oxygen atoms of carboxy groups. The hydrogen bonds are characterized by donor acceptor distances between $2.83 \AA$ and $2.95 \AA$ (hydrogen acceptor distances between $1.89 \AA$ and $2.06 \AA$ ) and $\mathrm{D}-\mathrm{H} \cdots \mathrm{O}$ angles between $165^{\circ}$ and $177^{\circ}$.

Table 1. Data collection and handling.

$\begin{array}{ll}\text { Crystal: } & \text { colorless prism, size } 0.24 \times 0.25 \times 0.26 \mathrm{~mm} \\ \text { Wavelength: } & \text { Mo } K_{\alpha} \text { radiation }(0.71073 \AA) \\ \mu: & 4.77 \mathrm{~cm}^{-1} \\ \text { Diffractometer, scan mode: } & \text { Nonius Kappa-CCD, } \Psi \\ 2 \theta_{\text {max: }} & 72.62^{\circ} \\ N(h k l)_{\text {measured }}, N(h k l)_{\text {unique: }} & 13108,13108 \\ \text { Criterion for } I_{\mathrm{obs}}, N(h k l)_{\mathrm{gl}}: & I_{\text {obs }}>2 \sigma\left(I_{\mathrm{obs}}\right), 12458 \\ N(\text { param })_{\text {refined: }} & 513 \\ \text { Programs: } & \text { SHELXS-97 [3], SHELXL-97 [4], } \\ & \text { ATOMS [5] }\end{array}$


Table 2. Atomic coordinates and displacement parameters (in $\AA^{2}$ ).

\begin{tabular}{llllll}
\hline Atom & Site & $x$ & $y$ & $z$ & $U_{\text {iso }}$ \\
\hline & & & & & \\
$\mathrm{H}(11 \mathrm{~A})$ & $4 a$ & $0.576(2)$ & $-0.225(1)$ & $1.0366(7)$ & $0.017(3)$ \\
$\mathrm{H}(11 \mathrm{~B})$ & $4 a$ & $0.599(2)$ & $-0.109(1)$ & $1.0252(7)$ & $0.016(3)$ \\
$\mathrm{H}(12 \mathrm{~A})$ & $4 a$ & $0.702(2)$ & $-0.100(1)$ & $0.9352(7)$ & $0.018(4)$ \\
$\mathrm{H}(12 \mathrm{~B})$ & $4 a$ & $0.715(1)$ & $-0.212(1)$ & $0.9122(7)$ & $0.018(3)$ \\
$\mathrm{H}(13 \mathrm{~A})$ & $4 a$ & $0.441(2)$ & $-0.309(1)$ & $0.9693(8)$ & $0.031(4)$ \\
$\mathrm{H}(13 \mathrm{~B})$ & $4 a$ & $0.559(2)$ & $-0.325(1)$ & $0.9184(8)$ & $0.025(4)$ \\
$\mathrm{H}(21 \mathrm{~A})$ & $4 a$ & $0.335(2)$ & $0.216(1)$ & $1.0079(7)$ & $0.017(4)$ \\
$\mathrm{H}(21 \mathrm{~B})$ & $4 a$ & $0.326(2)$ & $0.113(1)$ & $1.0443(7)$ & $0.019(4)$ \\
$\mathrm{H}(22 \mathrm{~A})$ & $4 a$ & $0.142(2)$ & $0.045(1)$ & $1.0252(8)$ & $0.029(4)$ \\
$\mathrm{H}(22 \mathrm{~B})$ & $4 a$ & $0.062(2)$ & $0.132(1)$ & $0.9869(7)$ & $0.020(4)$ \\
$\mathrm{H}(23 \mathrm{~A})$ & $4 a$ & $0.153(1)$ & $0.208(1)$ & $0.9032(7)$ & $0.014(3)$ \\
$\mathrm{H}(23 \mathrm{~B})$ & $4 a$ & $0.300(2)$ & $0.232(1)$ & $0.9048(8)$ & $0.024(4)$ \\
$\mathrm{H}(3 \mathrm{~A})$ & $4 a$ & $0.896(2)$ & $-0.159(1)$ & $0.8158(8)$ & $0.026(4)$ \\
$\mathrm{H}(3 \mathrm{~B})$ & $4 a$ & $0.958(2)$ & $-0.253(1)$ & $0.8438(8)$ & $0.030(4)$ \\
$\mathrm{H}(3 \mathrm{C})$ & $4 a$ & $1.027(2)$ & $-0.150(1)$ & $0.8515(9)$ & $0.034(5)$ \\
$\mathrm{H}(31 \mathrm{~A})$ & $4 a$ & $1.127(2)$ & $-0.254(1)$ & $0.7732(7)$ & $0.021(4)$ \\
$\mathrm{H}(31 \mathrm{~B})$ & $4 a$ & $0.992(2)$ & $-0.234(1)$ & $0.7330(8)$ & $0.025(4)$ \\
$\mathrm{H}(32 \mathrm{~A})$ & $4 a$ & $1.174(2)$ & $-0.077(1)$ & $0.7673(7)$ & $0.021(4)$ \\
$\mathrm{H}(32 \mathrm{~B})$ & $4 a$ & $1.157(2)$ & $-0.117(1)$ & $0.6964(8)$ & $0.032(4)$ \\
$\mathrm{H}(33 \mathrm{~A})$ & $4 a$ & $1.045(2)$ & $0.044(1)$ & $0.7178(8)$ & $0.029(4)$ \\
& & & & & \\
\hline
\end{tabular}

Table 2. Continued.

\begin{tabular}{llllll}
\hline Atom & Site & $x$ & $y$ & $z$ & \multicolumn{1}{l}{$U_{\text {iso }}$} \\
\hline H(33B) & $4 a$ & $0.948(1)$ & $-0.007(1)$ & $0.7647(7)$ & $0.012(3)$ \\
H(34A) & $4 a$ & $0.853(2)$ & $-0.112(1)$ & $0.6813(8)$ & $0.034(4)$ \\
H(34B) & $4 a$ & $0.841(2)$ & $0.009(1)$ & $0.6627(8)$ & $0.034(5)$ \\
H(34C) & $4 a$ & $0.959(2)$ & $-0.060(1)$ & $0.6289(8)$ & $0.032(4)$ \\
H(4A) & $4 a$ & $0.607(2)$ & $-0.046(1)$ & $0.7148(8)$ & $0.036(5)$ \\
H(4B) & $4 a$ & $0.601(2)$ & $-0.063(1)$ & $0.6450(8)$ & $0.034(4)$ \\
H(4C) & $4 a$ & $0.486(2)$ & $-0.015(1)$ & $0.6808(8)$ & $0.030(4)$ \\
H(41A) & $4 a$ & $0.449(2)$ & $-0.157(1)$ & $0.7385(8)$ & $0.024(4)$ \\
H(41B) & $4 a$ & $0.576(2)$ & $-0.211(1)$ & $0.7073(7)$ & $0.021(4)$ \\
H(42A) & $4 a$ & $0.332(1)$ & $-0.156(1)$ & $0.6401(7)$ & $0.015(3)$ \\
H(42B) & $4 a$ & $0.454(2)$ & $-0.215(1)$ & $0.6049(7)$ & $0.022(4)$ \\
H(43A) & $4 a$ & $0.318(1)$ & $-0.301(1)$ & $0.7179(7)$ & $0.016(4)$ \\
H(43B) & $4 a$ & $0.416(2)$ & $-0.364(1)$ & $0.6721(7)$ & $0.024(4)$ \\
H(44A) & $4 a$ & $0.153(2)$ & $-0.297(1)$ & $0.6288(7)$ & $0.028(4)$ \\
H(44B) & $4 a$ & $0.256(2)$ & $-0.357(1)$ & $0.5867(8)$ & $0.032(4)$ \\
H(44C) & $4 a$ & $0.191(2)$ & $-0.412(1)$ & $0.6462(8)$ & $0.031(4)$ \\
HW(1A) & $4 a$ & $0.698(2)$ & $-0.401(2)$ & $0.786(1)$ & $0.052(6)$ \\
HW(1B) & $4 a$ & $0.735(2)$ & $-0.412(1)$ & $0.8485(8)$ & $0.030(4)$ \\
HW(2A) & $4 a$ & $0.749(2)$ & $0.105(1)$ & $1.0417(8)$ & $0.030(4)$ \\
HW(2B) & $4 a$ & $0.857(2)$ & $0.043(1)$ & $1.0255(9)$ & $0.042(5)$ \\
& & & & & \\
\hline & & & & &
\end{tabular}

Table 3. Atomic coordinates and displacement parameters (in $\AA^{2}$ ).

\begin{tabular}{|c|c|c|c|c|c|c|c|c|c|c|}
\hline Atom & Site & $x$ & $y$ & $z$ & $U_{11}$ & $U_{22}$ & $U_{33}$ & $U_{12}$ & $U_{13}$ & $U_{23}$ \\
\hline $\operatorname{Zr}(1)$ & $4 a$ & $0.356687(8)$ & $-0.054974(6)$ & $0.907108(4)$ & $0.00714(3)$ & $0.00743(3)$ & $0.00785(3)$ & 0.00051 & $-0.00016(3)$ & $-0.00107(3)$ \\
\hline$N(1)$ & $4 a$ & $0.52864(8)$ & $-0.17550(7)$ & $0.94585(4)$ & $0.0108(3)$ & $0.0123(3)$ & $0.0101(3)$ & $0.0004(3)$ & $0.0006(3)$ & $0.0003(3)$ \\
\hline$N(2)$ & $4 a$ & $0.25277(8)$ & $0.10139(6)$ & $0.95403(4)$ & $0.0089(3)$ & $0.0116(3)$ & $0.0109(3)$ & $-0.0012(3)$ & $0.0005(3)$ & $-0.0028(3)$ \\
\hline$N(3)$ & $4 a$ & $0.97748(9)$ & $-0.19019(7)$ & $0.82359(4)$ & $0.0119(3)$ & $0.0148(4)$ & $0.0131(4)$ & $-0.0019(3)$ & $0.0001(3)$ & $0.0015(3)$ \\
\hline$N(4)$ & $4 a$ & $0.55303(9)$ & $-0.06285(8)$ & $0.68286(4)$ & $0.0155(4)$ & $0.0157(4)$ & $0.0163(4)$ & $-0.0015(3)$ & $-0.0023(3)$ & $0.0008(3)$ \\
\hline$C(11)$ & $4 a$ & $0.5408(1)$ & $-0.16257(8)$ & $1.01601(5)$ & $0.0109(4)$ & $0.0176(4)$ & $0.0109(4)$ & $0.0023(3)$ & $-0.0003(3)$ & $0.0006(3)$ \\
\hline$C(12)$ & $4 a$ & $0.65569(9)$ & $-0.15261(8)$ & $0.91262(5)$ & $0.0086(3)$ & $0.0185(4)$ & $0.0135(4)$ & $0.0017(3)$ & $0.0010(3)$ & $0.0018(3)$ \\
\hline$C(13)$ & $4 a$ & $0.4806(1)$ & $-0.28134(8)$ & $0.93040(5)$ & $0.0166(4)$ & $0.0095(4)$ & $0.0172(4)$ & $0.0020(3)$ & $-0.0003(4)$ & $0.0009(3)$ \\
\hline$C(14)$ & $4 a$ & $0.4066(1)$ & $-0.13377(7)$ & $1.04459(5)$ & $0.0127(4)$ & $0.0104(4)$ & $0.0116(4)$ & $-0.0002(3)$ & $0.0006(3)$ & $0.0008(3)$ \\
\hline$C(15)$ & $4 a$ & $0.6303(1)$ & $-0.10838(7)$ & $0.84641(4)$ & $0.0107(4)$ & $0.0112(4)$ & $0.0116(4)$ & $-0.0002(3)$ & $0.0014(3)$ & $-0.0023(3)$ \\
\hline$C(16)$ & $4 a$ & $0.3749(1)$ & $-0.28488(7)$ & $0.87807(5)$ & $0.0134(4)$ & $0.0106(4)$ & $0.0187(4)$ & $0.0003(3)$ & $0.0016(3)$ & $-0.0016(3)$ \\
\hline$C(21)$ & $4 a$ & $0.3439(1)$ & $0.14399(8)$ & $1.00312(5)$ & $0.0113(4)$ & $0.0166(4)$ & $0.0135(4)$ & $-0.0001(3)$ & $-0.0014(3)$ & $-0.0065(3)$ \\
\hline$C(22)$ & $4 a$ & $0.12348(9)$ & $0.07249(7)$ & $0.98263(5)$ & $0.0108(4)$ & $0.0139(4)$ & $0.0146(4)$ & $-0.0007(3)$ & $0.0037(3)$ & $-0.0036(3)$ \\
\hline$C(23)$ & $4 a$ & $0.2331(1)$ & $0.17668(7)$ & $0.90096(5)$ & $0.0173(4)$ & $0.0098(4)$ & $0.0156(4)$ & $0.0026(3)$ & $0.0018(4)$ & $-0.0006(3)$ \\
\hline$C(24)$ & $4 a$ & $0.4879(1)$ & $0.11732(7)$ & $0.98817(5)$ & $0.0120(4)$ & $0.0109(4)$ & $0.0109(4)$ & $-0.0002(3)$ & $-0.0004(3)$ & $-0.0009(3)$ \\
\hline$C(25)$ & $4 a$ & $0.05942(9)$ & $-0.01514(7)$ & $0.94609(5)$ & $0.0099(4)$ & $0.0117(4)$ & $0.0114(4)$ & $0.0009(3)$ & $-0.0005(3)$ & $0.0011(3)$ \\
\hline$C(26)$ & $4 a$ & $0.2509(1)$ & $0.13179(7)$ & $0.83434(5)$ & $0.0100(4)$ & $0.0108(4)$ & $0.0139(4)$ & $0.0004(3)$ & $-0.0002(3)$ & $-0.0003(3)$ \\
\hline$C(31)$ & $4 a$ & $1.0521(1)$ & $-0.20512(8)$ & $0.76227(5)$ & $0.0132(4)$ & $0.0178(4)$ & $0.0147(4)$ & $-0.0005(3)$ & $0.0015(3)$ & $-0.0017(4)$ \\
\hline $\mathrm{C}(32)$ & $4 a$ & $1.1050(1)$ & $-0.10305(9)$ & $0.73784(5)$ & $0.0122(4)$ & $0.0222(5)$ & $0.0147(4)$ & $-0.0043(3)$ & $0.0006(3)$ & $0.0001(4)$ \\
\hline$C(33)$ & $4 a$ & $0.9998(1)$ & $-0.02081(9)$ & $0.72540(5)$ & $0.0182(5)$ & $0.0164(4)$ & $0.0171(5)$ & $-0.0033(4)$ & $0.0027(4)$ & $0.0008(4)$ \\
\hline $\mathrm{C}(34)$ & $4 a$ & $0.9047(1)$ & $-0.0482(1)$ & $0.67116(6)$ & $0.0193(5)$ & $0.0289(6)$ & $0.0257(5)$ & $-0.0017(5)$ & $-0.0066(4)$ & $0.0049(5)$ \\
\hline$C(41)$ & $4 a$ & $0.4975(1)$ & $-0.16590(8)$ & $0.69932(5)$ & $0.0183(4)$ & $0.0131(4)$ & $0.0144(4)$ & $-0.0008(3)$ & $-0.0026(4)$ & $0.0009(3)$ \\
\hline $\mathrm{C}(42)$ & $4 a$ & $0.4056(1)$ & $-0.20854(8)$ & $0.64821(5)$ & $0.0143(4)$ & $0.0148(4)$ & $0.0121(4)$ & $-0.0003(3)$ & $0.0000(3)$ & $-0.0009(3)$ \\
\hline$C(43)$ & $4 a$ & $0.3452(1)$ & $-0.31061(8)$ & $0.67112(5)$ & $0.0161(4)$ & $0.0152(4)$ & $0.0147(4)$ & $-0.0010(4)$ & $0.0008(4)$ & $-0.0002(3)$ \\
\hline$C(44)$ & $4 a$ & $0.2287(1)$ & $-0.34672(9)$ & $0.63040(6)$ & $0.0200(5)$ & $0.0172(5)$ & $0.0204(5)$ & $-0.0041(4)$ & $0.0008(4)$ & $-0.0012(4)$ \\
\hline$O(14 A)$ & $4 a$ & $0.38826(7)$ & $-0.14178(6)$ & $1.10299(3)$ & $0.0189(3)$ & $0.0179(3)$ & $0.0097(3)$ & $0.0032(3)$ & $0.0020(2)$ & $0.0013(2)$ \\
\hline$O(14 B)$ & $4 a$ & $0.31899(7)$ & $-0.09855(6)$ & $1.00547(3)$ & $0.0107(3)$ & $0.0158(3)$ & $0.0113(3)$ & $0.0014(2)$ & $0.0006(2)$ & $0.0018(3)$ \\
\hline$O(15 A)$ & $4 a$ & $0.71983(7)$ & $-0.10561(6)$ & $0.80581(4)$ & $0.0120(3)$ & $0.0206(4)$ & $0.0137(3)$ & $0.0007(3)$ & $0.0039(3)$ & $0.0001(3)$ \\
\hline$O(15 B)$ & $4 a$ & $0.51359(7)$ & $-0.07160(6)$ & $0.83710(3)$ & $0.0116(3)$ & $0.0154(3)$ & $0.0109(3)$ & $0.0040(2)$ & $0.0013(2)$ & $-0.0007(2)$ \\
\hline$O(16 A)$ & $4 a$ & $0.3547(1)$ & $-0.36347(6)$ & $0.84829(5)$ & $0.0247(4)$ & $0.0132(3)$ & $0.0384(5)$ & $0.0025(3)$ & $-0.0075(4)$ & $-0.0110(3)$ \\
\hline$O(16 B)$ & $4 a$ & $0.30618(7)$ & $-0.20056(6)$ & $0.87170(4)$ & $0.0139(3)$ & $0.0102(3)$ & $0.0208(4)$ & $0.0020(2)$ & $-0.0034(3)$ & $-0.0035(3)$ \\
\hline$O(24 A)$ & $4 a$ & $0.57786(8)$ & $0.16422(6)$ & $1.01539(4)$ & $0.0133(3)$ & $0.0172(3)$ & $0.0188(3)$ & $-0.0017(3)$ & $-0.0036(3)$ & $-0.0060(3)$ \\
\hline $\mathrm{O}(24 \mathrm{~B})$ & $4 a$ & $0.50582(7)$ & $0.04157(5)$ & $0.94901(3)$ & $0.0105(3)$ & $0.0121(3)$ & $0.0130(3)$ & $-0.0003(2)$ & $0.0006(2)$ & $-0.0040(2)$ \\
\hline$O(25 A)$ & $4 a$ & $-0.05807(7)$ & $-0.03752(6)$ & $0.95570(4)$ & $0.0095(3)$ & $0.0181(4)$ & $0.0185(3)$ & $-0.0013(2)$ & $0.0020(3)$ & $-0.0017(3)$ \\
\hline$O(25 B)$ & $4 a$ & $0.13666(6)$ & $-0.06447(5)$ & $0.90744(3)$ & $0.0092(2)$ & $0.0136(3)$ & $0.0141(3)$ & $0.0009(2)$ & $-0.0003(3)$ & $-0.0037(3)$ \\
\hline$O(26 A)$ & $4 a$ & $0.21449(8)$ & $0.18048(6)$ & $0.78721(4)$ & $0.0241(4)$ & $0.0161(4)$ & $0.0163(3)$ & $0.0062(3)$ & $-0.0037(3)$ & $0.0025(3)$ \\
\hline$O(26 B)$ & $4 a$ & $0.31328(7)$ & $0.04523(6)$ & $0.83119(3)$ & $0.0153(3)$ & $0.0115(3)$ & $0.0116(3)$ & $0.0039(2)$ & $0.0002(2)$ & $-0.0001(3)$ \\
\hline OW(1) & $4 a$ & $0.67070(8)$ & $-0.42979(7)$ & $0.82355(4)$ & $0.0172(4)$ & $0.0334(5)$ & $0.0170(4)$ & $-0.0071(3)$ & $0.0010(3)$ & $-0.0011(3)$ \\
\hline OW(2) & $4 a$ & $0.82919(8)$ & $0.08416(7)$ & $1.05418(4)$ & $0.0151(4)$ & $0.0249(4)$ & $0.0173(3)$ & $0.0049(3)$ & $-0.0005(3)$ & $-0.0038(3)$ \\
\hline
\end{tabular}


Acknowledgments. The authors wish to thank the Austrian Fonds zur Förderung der wissenschaftlichen Forschung FWF (P11684-GEO) and the International Center for Diffraction Data for financial assistance of this work (Grant 90-03 ET).

\section{References}

1. Haussühl, E.; Haussühl, S.: Crystal structure, phase transitions, anomalous thermal and elastic properties of bis (butylammonium) zirconium bis(nitrilotriacetate) dihydrate and bis(butylammonium) hafnium bis(nitrilotriacetate) dihydrate, $\left[\mathrm{NH}_{3}\left(\mathrm{CH}_{2}\right)_{3} \mathrm{CH}_{3}\right]_{2} \mathrm{X}\left[\mathrm{N}\left(\mathrm{CH}_{2} \mathrm{COO}\right)_{3}\right]_{2}$ $2 \mathrm{H}_{2} \mathrm{O},(X=\mathrm{Zr}, \mathrm{Hf})$. Z. Kristallogr. 214 (1999), in press.
2. Hoard, J. L.; Silverton, E. W.; Silverton J. V.: Stereochemistry of Discrete Eight- Coordination. IV. The Structure of the Bis(nitrilotriacetato)zirconate (IV) Ion in Crystals of Potassium Salt. J. Amer. Chem. Soc. 90 (1968) 2300-2308.

3. Sheldrick, G. M.: SHELXS-97. Program for the solution of crystal structures. University of Göttingen, Göttingen, Germany 1997.

4. Sheldrick, G. M.: SHELXL-97. Program for Refinement of Crystal Structures. University of Göttingen, Germany 1997.

5. Dowty, E.: Atoms 3.2, A Complete Program for Displaying Atomic Structures. By Shape software, 521 Hidden Valley Road, Kingsport, TN 37663 , USA 1995. 